\title{
Printable Paper-Based Polymer Sensors for Detection of Vapor Phase Alcohols
}

\author{
John S. Mitchell', Richard Oliver ${ }^{1}$, Bob Jordan ${ }^{1}$ \\ ${ }^{1}$ Bioengineering Technologies Group, The New Zealand Institute for Plant \& Food Research Ltd, \\ Private Bag 3230, Hamilton, New Zealand \\ john.mitchell@plantandfood.co.nz
}

\begin{abstract}
:
Porous sol-gel polymer thin films doped with gold nanoparticles are coated onto paper substrates to serve as low-cost plasmonic gas sensors. Once the polymer is set, it can swell in response to the presence of organic volatiles, thus altering the inter-particle distance between nanoparticles and blueshifting the plasmon resonance peak. A sol-gel formulation is selected to achieve appropriate porosity and hydrophobicity for detection of the short chain alcohols methanol and ethanol. This polymer matrix surrounds the gold nanoparticles without causing aggregation and forms a thin film which interpenetrates the pores of the paper substrate. Plasmon shifts are detected by use of a fiber optic spectrometer combined with peak fitting algorithms to determine plasmon peak position accurately. Sensor responses were analyzed after passive exposure to alcohol vapors in sealed vessels. Limits of detection of $160 \mathrm{ppm}$ for methanol and $100 \mathrm{ppm}$ for ethanol were achieved, sufficient for use in industrial process control and safety applications. The plasmonic responses are reversible, making the sensors re-usable which, combined with low substrate costs, offers an inexpensive alcohol sensor.
\end{abstract}

Key words: polymer, plasmonic gas sensors, alcohols, gold

\section{Low-Cost Plasmonic Gas Sensing}

Chemical sensing of organic volatiles has tended to focus on electrochemical approaches, including carbon nanotube or graphenemodified sensors [1] and gas ionization detectors [2], or fast mass spectrometry [3]. There is a need for low-cost alternatives that do not require elaborate instrumentation to measure volatile organic compounds (VOCs), such as short chain alcohols, at concentrations typically encountered in industrial processes. Optical sensors that utilize low-cost substrates, such as paper, could offer a means of achieving organic volatiles detection affordably across a large number of locations by using small tags which, in future, could be read by a convenient hand-held reader device. Such sensor tags, if of low cost, could be made disposable. However, if they are re-usable this could make them even more affordable. In many applications, specificity is also not an issue, as only a total VOC determination is needed.

A number of solution phase DNA-based sensors [4] have harnessed changes in the inter-particle distance of gold nanoparticles and the concomitant changes in plasmonic peak position [5]. Such an approach could also be applied to gas sensing when the gold nanoparticles are doped into a polymer matrix that swells in response to certain VOCs. Silicon-based sol-gels offer a porous crosslinked network which can swell to a limited extent in response to VOCs whilst maintaining comparatively low thermosensitivity [6]. They can also be easily printed onto a variety of substrates. This combination could form the basis for a convenient and low-cost gas sensor technology.

\section{Polymer Composite Coating and Reading of Sensor Tags}

A porous sol-gel polymer thin film doped with $20 \mathrm{~nm}$ gold nanoparticles (supplied by BBI with size control by Transmission Electron Microscopy) was drop-coated onto $6 \mathrm{~mm} \times 6$ $\mathrm{mm}$ squares of filter paper. The proportions of tetraethyl orthosilicate (TEOS) monomer, water, and the gold nanoparticle concentration were optimized to give a clear red color with minimized aggregation of the gold colloid upon mixing and polymer setting. The nanoparticles were compatible with the polymer matrix and once the polymer was set, they remained unaggregated. The optimized formulation allowed a relatively even color to form across the support matrix after setting, and the porosity of the filter paper was essential in stabilizing the 
polymer and allowing even distribution of nanoparticles. The polymerization was acidcatalyzed, with an initial setting complete within $1 \mathrm{~h}$ and complete setting within 1 week. As the color of the sensors is generated by gold nanoparticles, the tags are not subject to photobleaching and so can be used in bright light environments.

The resulting sensor tags were interrogated by a Zeiss spectrometer via a fiber optic which supplied the incident light and guided the reflected light to the spectrometer. A typical example of the spectrum acquired from the sensors is given in Figure 1, along with an enlargement showing a typical plasmon response. The spectra were collected using a LABView $^{\text {TM }}$ interface at 10 different positions across the sensor taken at random. The spectrometer was re-calibrated against an internal white reference before every measurement. The data were tabulated in a spreadsheet format automatically.

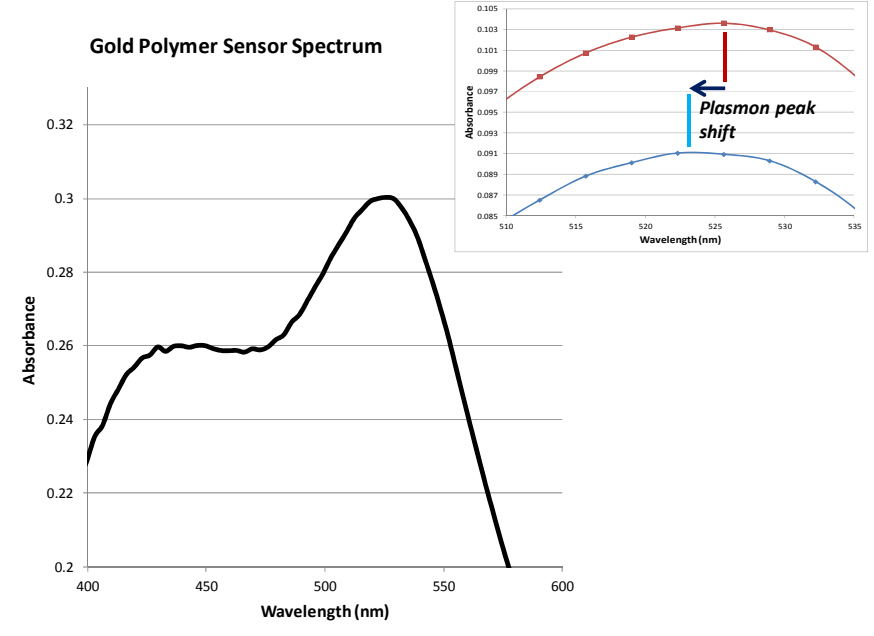

Fig. 1. The spectrum of the gold-doped printed polymer sensor, with the plasmon peak shift illustrated for exposure to methanol vapor (inset)

The plasmon peak position was determined by using a quadratic peak-fitting algorithm between pre-defined wavelength bounds (typically 510$550 \mathrm{~nm}$ ). The peak positions were averaged across the 10 surface locations. This analysis was performed both before and after gas exposure.

The concentration of gold nanoparticles within the polymer matrix could be closely controlled and was varied to achieve plasmon absorbances between 0.08 and 0.31 . Examples of the sensor tags at both high and low gold concentrations are given in Figure 2. In general, lower gold concentrations were found to give a more sensitive response. The starting wavelengths of the plasmon peaks also changed with nanoparticle concentration between approximately 515 and $530 \mathrm{~nm}$. Sufficient setting time for the sol-gel polymer was needed to stabilize the starting wavelength ready for alcohol sensing.

\section{Gas Exposure and Sensor Responses}

The sensors were placed into sealed plastic vessels $(450 \mathrm{~mL})$ which had been previously evaluated to ensure they did not release organic volatiles and were gastight. Methanol and ethanol vapors were separately introduced via injection of headspace samples through septa. The headspace samples were generated by using methanol or ethanol mixed with water in separate vials to generate a known headspace concentration according to Raoult's law. Vapor concentrations were further confirmed by gas chromatography with flame ionization detection (GC-FID). After exposure to alcohol vapors, the sensors were removed from the gas vessels and read immediately, and then at set times after venting (typically $1 \mathrm{~h}$ and $3 \mathrm{~h}$ post exposure). The sensors gave plasmon shifts of between 0.1 and $1.5 \mathrm{~nm}$. The sensors were tested against methanol and ethanol, both of which could produce plasmon shifts. Exposure to relative humidity up to $70 \%$ did not cause a clear swelling response in the plasmon peaks.

The labels typically required $4 \mathrm{~h}$ of alcohol exposure to achieve optimal response. The time taken to respond to the alcohol vapors is probably related to the pore size of the polymer which has been deliberately restricted to favor penetration by smaller gas molecules. The plasmon peak position relaxed back over 3-6 $\mathrm{h}$ 
after removal from the gas atmosphere, thus making them self-regenerating and re-usable sensors, Figure 2. The plasmon shift of the sensors should therefore reflect the concentrations to which they have most recently been exposed, and so the sensors could give a near-real-time concentration determination.

The sensors demonstrated limits of detection (LOD) of $160 \mathrm{ppm}$ for methanol and 100 ppm for ethanol, although further testing and optimization may further lower the LOD. The exact mechanism by which the response occurs is not clearly known, although it is probably due to polymer swelling even though silica-based sol-gels are known to swell less than polymers such as polyurethane foams. This difference in swelling response has the advantage that the sol-gel is expected to be less temperature sensitive than other polymer alternatives. The sensors do not possess total selectivity for methanol and ethanol; however, control of polymer porosity and the comparative hydrophobicity of the coating minimize potential interferences. The sensors would be used in environments where methanol and/or ethanol are the predominant volatile organic compounds, or else the sensors could be used in combination with selective membranes or outer films to block potentially interfering compounds.

It is envisaged that the labels could in future be read using a handheld reader device with which the plasmon peak shifts and corresponding gas concentrations may be determined automatically. Some partial selectivity can be designed into the sensors by altering the pore structure and hydrophobicity of the polymer. The low cost of the substrate, low gold loading (as the gold is in colloidal form), convenient optical data download and re-usability of the labels make them useful as potential vapor sensors for on-site industrial monitoring or process control, where a network of such sensors could be implemented.

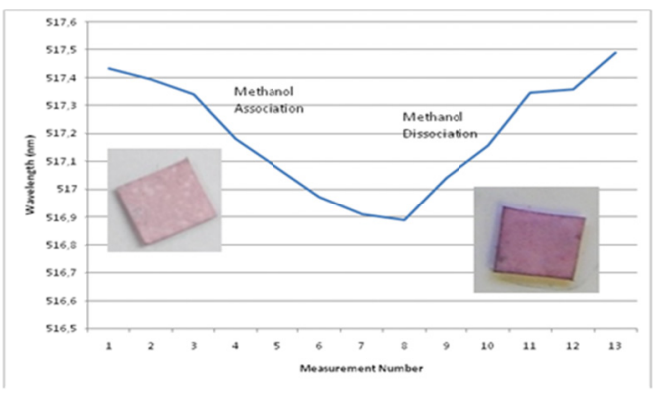

Fig. 2. A wavelength response plot for 180 ppm methanol vapor association followed by dissociation over time. Gold-doped nanoparticle sensors are shown also (inset).

\section{Conclusions}

A sensor for methanol and ethanol vapors has been constructed by doping gold nanoparticles into a silica-based sol-gel polymer thin film coated onto a porous paper substrate. Gas concentrations are determined by a shift in the plasmon peak position of the interrogated surface of the sensor resulting from a change in the inter-particle distance. These sensors are re-usable, reflect gas concentrations in nearreal-time, are low-cost and can detect alcohol concentrations typically found in industrial processes.

\section{Acknowledgements}

The authors thank the New Zealand Ministry of Science and Innovation (Project: C11X1003) for funding this work.

\section{References}

[1] T. Kuila, S. Bose, P. Khanra, A. Mishra, N. Kim, J. Lee, Recent Advances in Graphene-Based Biosensors, Biosensors \& Bioelectronics 26, 4637-4648 (2011); doi: 10.1016/j.bios.2011.05.039

[2] L. Velasquez-Garcia, B. Gassend, A. Akinwande, CNT-Based MEMS/NEMS Gas lonizers for Portable Mass Spectrometry Applications, Journal of Microelectromechanical Systems 19, 484-493 (2010); doi: 10.1109/JMEMS.2010.2045639

[3] K. Pratt, K. Prather, Mass Spectrometry of Atmospheric Aerosols: Recent Developments and Applications, Mass Spectrometry Reviews 31, 17-48 (2012); doi: 10.1002/mas.20330

[4] R. Elghanian, J. Storhoff, R. Mucic, R. Letsinger, C. Mirkin, Selective Colorimetric Detection of Polynucleotides Based on the DistanceDependent Optical Properties of Gold Nanoparticles, Science 277, 1078-1081 (1997); doi:10.1126/science.277.5329.1078

[5] W. Rechberger, A. Holhenau, A. Leitner, J. Krenn., B. Lamprecht, F. Aussenegg, Optical Properties of Two Interacting Gold Nanoparticles, Optics Communications 220, 137-141 (2003); doi: 10.1016/S0030-4018(03)01357-9

[6] W. Huang, W. Lee, Effect of TEOS Contents on Swelling Behaviors and Mechanical Properties of Thermosensitive Hybrid Gels Polymer Composites 31, 887-896 (2010); doi: 10.1002/pc.20871 